\title{
A synthetic model gut system to study macronutrient digestion in vitro
}

\author{
P. I. Chater, M. Wilcox, I. Brownlee and J. P. Pearson \\ ICaMB, Newcastle Medical School, Newcastle University, NE1 7RU
}

The digestion and absorption of macronutrients (fat, protein and carbohydrate) is a major factor in health and metabolic disease. By 2015 it is projected that $2 \cdot 3$ billion people will be overweight and 700 million obese ${ }^{(1)}$. Obesity alone affects more than $25 \%$ of the adult population costing the NHS over $£ 5$ billion per year ${ }^{(2)}$. Modulating macronutrient digestion with food additives and pharmaceuticals has been shown to be a fruitful approach to the treatment of obesity (Orlistat) ${ }^{(3)}$ and diabetes (Acarbose) ${ }^{(4)}$. Previous work has shown that bioactive agents have novel modulatory affects on the major enzymes of digestion ${ }^{(5)}$. Work in this lab has shown that specific alginates can inhibit pancreatic lipase up to $70 \%$, this is now being investigated as a potential anti-obesity agent ${ }^{(6)}$.

A model gut system which provides a physiologically relevant in vitro simulation of the digestive tract was developed to simulate the gastrointestinal tract from mouth to terminal small intestine capable of providing a continuous profile of digestion of whole foods, substrates, therapeutics, delivery systems and their effects on digestion. The model gut system (MGS) consists of a temperature and $\mathrm{pH}$ controlled system with compartments that accurately model physiological amounts and concentrations of enzymes, digestive secretions and cofactors.

The model gut system was validated using known enzyme inhibitors. At $\mathrm{T}_{[60]}$ by the end of the gastric phase, pentosan polysulphate significantly inhibited the digestion of protein by $87.6 \%$. At $\mathrm{T}[180]$ by the end of the small-intestinal phase protein digestion was completely inhibited by Soybean Trypsin Inhibitor. With Orlistat (10 mg/ml), fat digestion was reduced by $99 \cdot 1 \%$. With $100 \mathrm{mg}$ Acarbose, by $\mathrm{T}[180]$ carbohydrate digestion was inhibited by $98 \cdot 55 \%$. Full time-course data is available for all inhibitors.

A number of novel regulatory effects have been demonstrated with seaweed biopolymers being studied in this lab. Alginate showed dose dependent inhibition of fat digestion, with a maximum inhibition of $58.3 \pm 22 \cdot 2 \%(p=0.018)$ at $3 \mathrm{mg} / \mathrm{ml}$ at the end of the small intestinal phase. Alginate reduced protein digestion by 50.5\% $(P=0.001)$ at the end of the gastric phase T[60]. Alginate reduced carbohydrate digestion in the first 15 minutes of the small intestinal phase by $89.3 \%(P=0.025)$, after which digestion recovered to control levels. Fucoidan reduced protein digestion at T[60], by $59.1 \%(P=0.0274)$ at $6 \mathrm{mg} / \mathrm{ml}$. In the first 15 minutes of the small intestinal phase, carbohydrate was reduced by $81.87 \%$ by fucoidan. Further regulatory effects of seaweeds and seaweed extracts have been demonstrated in microplate assays, ethanol extracts of both fresh and dried Malaysian Red Seaweed showed potent inhibition of lipase activity, up to $92 \%$ and significant inhibition of $\alpha$-amylase by $88 \%$.

Conclusions: The model provides a robust, cost effective and ethical alternative to animal models or human studies and provides a tool for the investigation of novel therapeutics and food additives for treatment of metabolic diseases such as obesity, diabetes and pancreatic insufficiency.

1. World Health Organization. Obesity and Overweight Statistics (2006). Geneva:WHO.

2. Department of Health. Programme Budgeting Guidance 2006-07. (2008) London: Department of Health.

3. Lucas KH, et al. (2001). Ann. Pharmacother. 35, 314-328.

4. Martin A.E. and Montgomery P.A (1996). Am. J. Health Syst. Pharm. 53, 2277-90.

5. Balasubramaniam V et al. (2013) J. Appl Phycol.

6. Wilcox et al. (2014) Food Chemistry, 146, 479-484 